\title{
Extending the Role of Analogies in the Teaching of Physics
}

Nikolaos Fotou and Ian Abrahams, University of Lincoln, Lincoln, England

$\mathrm{R}$ esearch in physics teaching has supported the use of analogies as an effective instructional tool that can be used to facilitate students' understanding of physics concepts. The effectiveness of analogies lies in that they allow students to form cognitive links between what they already know and what they are learning, harmoniously integrating, in this way, the new physics concepts into their existing knowledge. In this paper, it is suggested that analogies could be extended to provide physics teachers with a diagnostic form of assessment that can reveal both the misconceptions their students may hold, the prior knowledge upon which such misconceptions are based, as well as knowledge sources that can be productively used in the teaching process. This suggestion arises from the findings of a cross-age study in which students, from five different age groups, were asked to make predictions about a range of situations they had not previously encountered (novel situations) and explain the reasons that led them to make those predictions.

\section{The didactic purpose of analogies in teaching physics}

Reasoning on the basis of analogies is a process whereby a known object or situation (base domain) is compared with an unknown one (target domain). The known base domain is used as the basis to approach the target that is under examination, with similarities being drawn from the former as a means to understand the latter (Fig. 1).

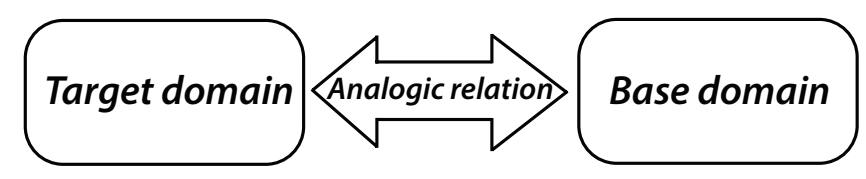

Fig. 1. The process of analogical reasoning.

In such an analogical relation, each of the two domains has certain relationships, or attributes, that can be mapped and transferred from the one domain to the other. An example of attributes is the well-discussed hydraulic analogy for electric current, ${ }^{1,2}$ with one of the similarities in their attributes being that water, like electricity, flows. Similarly, an example of relationship mapping can be seen in the planetary model for the atomic structure whereby the relationship between the electrons that orbit the nucleus are seen as being similar to the relationship between the planets and the Sun that they orbit.

Different terms have been used for relationships or attributes mapping, amongst which are these of literal similarities, similes, examples, and models, without, however, a clear distinction between them and that of analogy. ${ }^{3}$ Whether something is an analogy, literal similarity, or whatever the term used seems to be less relevant than the reasoning as a process itself that is employed in an attempt to perceive an unfamiliar situation on the basis of something familiar. It might be ar- gued, even within the study reported here, that there could be some cases in which the analogies generated are considered more akin to examples or similes, but the focus is on whether there is transfer of knowledge from one situation to another regardless of how this process is termed.

Within a constructivist approach, students actively construct new meanings on the basis of their existing knowledge, and the advantages of analogies in that process is that they open new perspectives by pointing to similarities between the abstract and unfamiliar with the real world or situations students are already familiar with. ${ }^{4}$ Similarly, the teaching of physics could be described as a process of making the unfamiliar familiar, with new knowledge being built upon prior understandings and experiences. ${ }^{3}$

Prior research has added instructional value to the use of analogies in physics for such didactical purposes, ${ }^{1,2}$ i.e., as instructional tools used for the introduction of a new concept, whereby students are provided with a ready-made analogy and asked to use it to understand the new, unfamiliar concept. Research ${ }^{5,6}$ has also indicated that analogies high school ${ }^{5}$ and university ${ }^{7}$ students self-generate might serve as a diagnostic form of assessment, thus revealing any ideas inconsistent with the scientific account that might be held-ideas that have been widely referred to in the literature as misconceptions. Here, by drawing on a wider age span, it is suggested that analogies students spontaneously self-generate, as opposed to situations where analogies are used for didactic purposes or situations where students' self-generation is prompted - students are explicitly asked to generate analogies- cannot only serve as a diagnostic form of misconceptions, but can also reveal both the sources upon which such misconceptions are founded, as well as their reasoning processes when they encounter an unfamiliar situation, concept, or phenomenon.

\section{The novel situations}

The paper builds on previous research ${ }^{8}$ in which students from five different age groups (Table I) were presented with six novel situations and were asked to make predictions about the outcome of a future event (effectively what would happen in the event depicted in the novel situation), and then write what led them to their predictions. The novel situations were presented in a pictorial form (Figs. 2 and 3) so as to be accessible across such a wide age range while devices such as "switching on bulbs" and "numbered persons" were used to avoid providing any kind of lead in terms of the selection of one particular option from those listed in the accompanying multiple-choice question, as well as to encourage students to articulate their thinking during their prediction-making process. The concepts involved in these six novel situations had been drawn from previous research on misconceptions in physics and chemistry. Here, two of these six novel situations in which physics concepts were involved, and the analogies students 
Table I. Students' predictions in the two novel situations and spontaneous generation of analogies per age group in the most prevalent answer.

\begin{tabular}{|c|c|c|c|c|c|c|}
\hline \multirow[b]{2}{*}{ 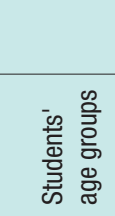 } & \multicolumn{3}{|c|}{ Predictions in weight and gravity novel situation } & \multicolumn{3}{|c|}{$\begin{array}{c}\text { Predictions in firing a cannon in a uniformly } \\
\text { moving boat novel situation }\end{array}$} \\
\hline & $\begin{array}{l}\text { A) Bulbs will } \\
\text { light up at the } \\
\text { same time }\end{array}$ & $\begin{array}{l}\text { B) Bulb A } \\
\text { will be first }\end{array}$ & $\begin{array}{l}\text { C) Bulb B } \\
\text { will be first }\end{array}$ & $\begin{array}{l}\text { A) Person } \\
\text { B }\end{array}$ & $\begin{array}{c}\text { B) Person } \\
\text { A }\end{array}$ & $\begin{array}{c}\text { C) Person } \\
\text { C }\end{array}$ \\
\hline $9-10$ & 0 & $36(20)$ & $1(1)$ & 0 & 0 & $37(15)$ \\
\hline $11-12$ & $2(N)$ & $28(19)$ & $1(1)$ & 0 & 0 & $31(12)$ \\
\hline $12-13$ & $3(N)$ & $26(13)$ & 0 & 0 & $1(1)$ & $28(12)$ \\
\hline $14-15$ & $8(4)$ & $27(17)$ & 0 & $3(2)$ & 0 & $32(11)$ \\
\hline $16-17$ & $7(4)$ & $27(12)$ & 0 & $4(3)$ & 0 & $30(12)$ \\
\hline
\end{tabular}

Note: Number in the parenthesis indicates spontaneous generation of analogies, whereas $N$ denotes no instances of analogy generation for these predictions made.

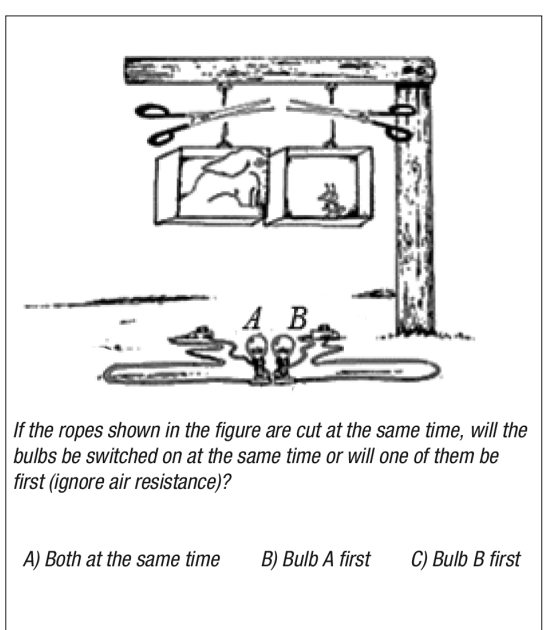

Fig. 2. Weight and gravity novel situation.

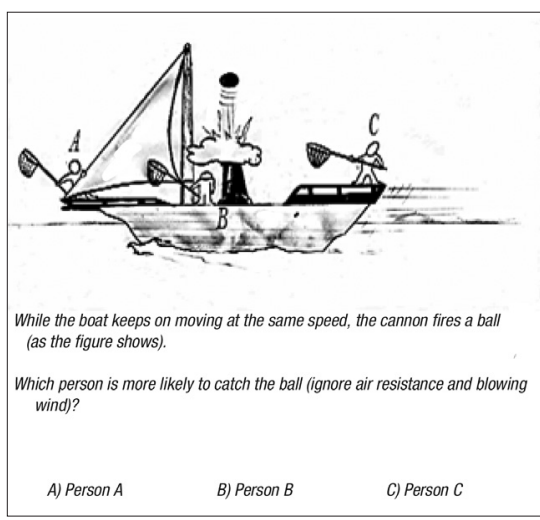

Fig. 3. Firing a cannon in a uniformly moving boat novel situation. ated while explaining their predictions, are examined.

\section{Results}

Analysis of students' responses showed that, in many cases, they spontaneously self-generated analogies in order to familiarize themselves with these two novel situations and be able to make, and subsequently explain, their prediction. The incorrect predictions students made were those that might have been expected based on the existing literature on students' misconceptions that the novel situations were set out to probe.

Consider, for example, the following response given by a 17-year-old student in the weight and gravity situation, which was set out to probe students' understanding of gravity and the role of weight in falling objects:

"I think this is like when you have a ball and a feather. I have seen a ball falling faster on the ground than a feather. I have answered that the box with the elephant in it will fall faster, since the weight in it is greater and there is a greater force in that box than in the other one with the ant in it. The heavier always goes faster as in the case with the feather and the ball." spontaneously gener-
The above example illustrates how students typically made predictions by reasoning on the basis of spontaneously generated analogies that were drawn from what they perceived as being similar and, in many cases, familiar everyday life experiences. Indeed, students from a very young age had seen objects of different mass, like bricks, pebbles, stones, and pieces of paper (to name but a few of the analogies students spontaneously generated), which were left to fall from the same height and were, they reported, observed to reach the ground in different times (the heavier always reaching the ground first). In these analogies, students articulated a rather common misconception according to which the heaviness is the reason for falling, and thus heavier objects precede lighter ones when falling.

Similarly, in the novel situation depicted in Fig. 2, the students, again often drawing on spontaneous analogies, articulated a common misunderstanding of simultaneous horizontal and vertical motion. Consider, for example, the following response given by a 15-year-old when explaining the most frequent prediction, namely that it would be person $\mathrm{C}$ who would catch the cannonball:

"It [the cannonball] will go upwards and it will fall back down. I think that if the ship was not moving, it would be the person in point $\mathrm{B}$ more likely to catch it but the question says that the ship is moving. So, the cannonball after going up it will fall down. Meanwhile, the ship has moved forward and the person in place $\mathrm{C}$ will be the one to catch the cannonball... It is like when I toss a ball up high. When I move forward fast, it will not come with me it will rather fall behind[sic]."

As the response illustrates, the understanding that the cannonball will fall straight down whilst the boat moves beneath it occurs when students observe, from afar, objects being dropped from a moving body. This is in contrast to when they themselves may drop an object they are carrying whilst moving, like, for example, a tennis ball dropped by a walking person or a marble being released by a person in a moving car (to name a few of the analogies students spontaneously generated). In those situations students reported that the object would still fall in a straight vertical path relative to the carrier because the latter usually acts as their frame of reference. The cannonball having been fired vertically into the air, whilst simultaneously being located on a moving boat, was reported by students to be a confusing and complex situation. Initially many of them thought about the situation as if the boat was stationary, generating the correct prediction of person B. However, they then rejected this solution in order to take account of the movement of the boat and the fact that they 
saw the cannonball having no horizontal impetus (unlike the boat), and so very often revised their predication to person $\mathrm{C}$.

Nevertheless, there were few cases, as Table I shows, in which students' spontaneous analogical reasoning facilitated the understanding of this novel situation and led them to the scientifically correct prediction. An example of such reasoning is the explanation given by a 15 -year-old student below:

"I have chosen that person B will catch the ball because when the cannonball is fired it goes up high while the ship is moving when fired and keeps on moving and thus it falls back to where it was fired from, just like when I was on the ferry boat the other day and I tossed my cell phone up. It went up high and while both the ferry and I on it were moving, it landed straight back onto the palm of my hand."

Students' responses showed that their prior knowledge influenced how they understood the novel situations, as well as the analogies they then spontaneously generated in order to make their predictions. From the examples above it can be seen that their spontaneously generated analogies revealed not only the misconceptions they held but also, in many cases, the origins of those misconceptions and the specific everyday familiar life experiences on which these were based. This analogical reasoning led most of them to make a prediction incompatible with the scientific account, but there were also cases in which the spontaneously generated analogies were effective in facilitating the understanding of the novel situations and helping them reach a conclusion/prediction compatible with the scientific account.

\section{Discussion}

In agreement with previous studies on self-generated analogies, ${ }^{7,8}$ the study showed that spontaneous analogies and reasoning in novel situations can also serve as a diagnostic form of assessment revealing students' pre-instructional knowledge that can sometimes give rise to misconceptions. Furthermore, the study showed that the use of students' spontaneously generated analogies as a diagnostic form of assessment can reveal the sources of such prior knowledge, and provide the physics teacher with an understanding of the ways in which students apply such familiar, real-world, experiential knowledge in situations with which they are unfamiliar.

The results also indicated that spontaneously generated analogies, although frequently leading to erroneous predictions and misunderstandings, do have the potential, in some situations, to lead to scientifically compatible predictions. Thus, such a diagnostic form of assessment could also provide teachers with valuable information about students' experienc- es and understanding that could be productively used in the teaching process, serving as a starting place for the introduction of new physics concepts.

\section{References}

1. T. Greenslade, "The hydraulic analogy for electric current," Phys. Teach. 41, 464-466 (Nov. 2003).

2. N. Fotou and I. Abrahams, "Doing with ideas: The role of talk in effective practical work in science," School Sci. Rev. 97 (359), 25-30 (2015).

3 . R. Duit, "On the role of analogies and metaphors in learning science," Sci. Educ. 75 (6), 649-672 (1991).

4. J. L. Lemke, Talking Science: Language, Learning, and Values (Ablex, Norwood, NJ, 1990).

5. N. B. Mozzer and R. Justi, "Students' pre- and post-teaching analogical reasoning when they draw their analogies," Int. J. Sci. Educ. 34 (3), 429-458 (2012).

6. K. M. Pittman, "Student-generated analogies: Another way of knowing?” J. Res. Sci. Teach. 36 (1), 1-22 (1999).

7. E. D. Wong, "Self-generated analogies as a tool for constructing and evaluating explanations of scientific phenomena," J. Res. Sci. Teach. 30 (4), 367-380 (1993).

8. N. Fotou and I. Abrahams, "On the Role of Analogies Beyond Their Didactic Purpose," in New Perspectives in Science Education Conference Proceedings (libreriauniversitaria, Florence, Italy, 2018), pp. 436-441.

Nikolaos Fotou is a senior lecturer in the School of Education at the University of Lincoln, UK, responsible for the MA in education programme. Prior to joining the University of Lincoln, he was a science education lecturer in Maynooth University, Ireland. He has studied physics at the University of Crete in Greece and obtained his MA and PhD degree in science education at the University of Leeds, UK. He has previously worked as a physics and mathematics private tutor and supply teacher, teaching from primary, secondary and tertiary education in Greece to undergraduate and postgraduate programmes in Ireland and the UK. He is particularly keen to foster a more evidence-based, as opposed to opinion-based, approach to educational initiatives and his research interests are in science, physics and mathematics education with a focus on analogical reasoning, self-generation of analogies, nature of students' knowledge, teaching and learning in the context of interactive pedagogies, by the use of ICT and design and evaluation of educational interventions. NFotou@lincoln.ac.uk

Ian Abrahams is head of the School of Education and Professor of Science Education at the University of Lincoln, UK. Previously a physics teacher he has, since graduating in 2007 with a PhD in Education from the University of York under the supervision of Professor Robin Millar, worked at the IOE (now UCL Institute of Education), the University of York, and the University Leeds before moving to the University of Lincoln in 2015. lan's research interests relate to practical work, misconceptions, Continuing Professional Development - in particular the effectiveness of such programmes - and paired mentoring, and this research has led him to collaborate with colleagues from China, Germany, Ireland, Israel, Singapore, South Korea and Turkey. lan was elected a Fellow of the Institute of Physics in 2017. 